\title{
Expertise effects on the perceptual and cognitive tasks of indoor rock climbing
}

\author{
Mirinda M. Whitaker ${ }^{1} \cdot$ Grant D. Pointon $^{1} \cdot$ Margaret R. Tarampi $^{2} \cdot$ Kristina M. Rand $^{1}$
}

Published online: 30 October 2019

(C) The Psychonomic Society, Inc. 2019

\begin{abstract}
Experts' cognitive abilities adapt in response to the challenges they face in order to produce elite-level performance. Expert athletes, in particular, must integrate their motor capabilities with their cognitive and perceptual processes. Indoor rock climbers are particularly unique athletes in that much of the challenge they face is to accurately perceive and consolidate multiple movements into manageable action plans. In the current study, we investigated how climbers' level of expertise influenced their perception of action capabilities, visual memory of holds, and memory of planned and performed motor sequences. In Experiment 1, climbers judged their perceived capability to perform single climbing moves and then attempted each movement. Skilled climbers were less confident, but perceived their action capabilities more accurately than less skilled climbers. In Experiment 2, climbers recalled holds on a route, as well as predicted and recalled move sequences before and after climbing, respectively. Expertise was positively associated with visual memory performance as well as planned and recalled motor sequence accuracy. Together, these findings contribute to our knowledge of motor expertise and suggest that motor expert's ability to accurately estimate their action capabilities may underlie complex cognitive processes in their domain of expertise.
\end{abstract}

Keywords Sequence memory $\cdot$ Skill acquisition $\cdot$ Perceptual judgment $\cdot$ Motor control $\cdot$ Cognitive expertise

Sports require athletes to dynamically respond to opponents, teammates, or objects in the environment. Many sports are open skill in that they are fast paced, reactive, and do not allow time for athletes to plan action sequences (Allard \& Starkes, 1991). However, some sports are closed skill, where the conditions required to achieve an end goal are stable, allowing athletes to form complex preplanned motor sequences (Poulton, 1957). Indoor rock climbing is a prime example of a sport where athletes plan action sequences to navigate routes filled with diverse arrays of hand and foot holds. Climbing is a unique domain for the study of perceptual and cognitive skills because climbers integrate complex yet stable arrangements of environmental properties (i.e., climbing holds) in similar ways to mind sports such as chess, go, and bridge, where planned action and memory have been examined in experts (Chase \& Simon, 1973; Engle \& Bukstel, 1978; Reitman, 1976). Despite the stable environment in which a climber acts upon,

Mirinda M. Whitaker

mirinda.whitaker@utah.edu

1 University of Utah, Salt Lake City, UT, USA

2 University of Hartford, West Hatford, CT, USA a climber's movement through the space (particularly on novel routes) is thought to be highly dynamic (Wright, Pinyan, Wickens, Keller, \& McLaughlin, 2018). Climbers are unique in that they perform the aforementioned cognitive tasks while engaging in an athletic activity where they must account for their body and physical capabilities in relation to environmental aspects.

Research from other motor expertise domains suggests that climbers would develop advantageous perceptual and cognitive skills as a function of their expertise (Allard, Graham, \& Paarsalu, 1980; Correia, Araújo, Cummins, \& Craig, 2012; Farrow \& Abernethy, 2003; Weast, Shockley, \& Riley, 2011). For example, experienced rugby, tennis, and basketball players perceive and judge domain-specific action capabilities more accurately than novice players or nonplayers do (Correia et al., 2012; Farrow \& Abernethy, 2003; Weast et al., 2011). Experts in mind sports such as chess and bridge, and refereed sports such as snooker, basketball, and field hockey, demonstrate better memory recall for structured game arrays (Abernethy, Neal, \& Koning, 1994; Allard et al., 1980; Charness, 1979; Chase \& Simon, 1973; Garland \& Barry, 1991; Reitman, 1976; Starkes, 1987; Ward \& Williams, 2003; Williams, Davids, Burwitz, \& Williams, 1993). Together, this literature suggests that expert climbers would 
have greater perceptual accuracy of action possibilities and superior cognitive abilities.

The first perceptual task climbers face is to accurately perceive their action capabilities (or affordances) in a climbing environment. Gibson (1979) defined affordances as the relationship between environmental properties and properties of the observer that specifies a behavior (e.g., a climbing move). Specifically, climbers must identify crucial properties of the climbing wall (e.g., angle and height) and holds (e.g., texture, shape, slant, size) relative to their physical constraints (e.g., hand size, leg length, wingspan) and capabilities (e.g., strength, flexibility). Pijper's and Bakker's (1993) work supports the idea that climbers show enhanced perception of general action capabilities, as climbers more accurately perceived their maximum overhead reaching capability compared with nonclimbers. Some have claimed that such improved perceptual abilities may underlie superior cognitive abilities in climbers, like improved visual memory for holds on routes (Boschker, Bakker, \& Michaels, 2002; Pezzulo, Barca, Bocconi, \& Borghi, 2010). However, reaching judgments differ from true climbing affordances, which are far more complex. It has yet to be tested whether climbing expertise would improve the perception of actual climbing affordances.

Perception of single moves (i.e., movements from one hold to another) is important, but also critical for successful climbing is the ability to perceive how consecutive moves can be linked together into an effective motor sequence plan prior to a climb. This requires climbers to integrate action capability judgments with cognitive processes that link those actions together into a motor sequence. Furthermore, it is important that climbers remember what sequences they performed in previous climbing attempts so that they can update or modify their action plans to successfully climb. It is advantageous for climbers to plan and remember these motor sequences before they climb because the attentional demands to maintain grip, balance, and body position make it difficult to overhaul motor sequence plans (Green, Draper, \& Helton, 2014; Green \& Helton, 2011). This task is difficult given that climbing routes typically require more moves than individuals' short-term memory capacities (Cowan, 2010; Ma, Husain, \& Bays, 2014; Miller, 1956). Given the task difficulty, climbers likely develop cognitive strategies that allow them to overcome the large amount of information they must process in order to plan motor sequences.

Previous research demonstrates cognitive advantages in expert rock climbers via their enhanced visual memory for holds in a climbing environment (Boschker et al., 2002; Pezzulo et al., 2010). Specifically, in an artificial experimental setting, experts (compared with nonexperts) were able to more accurately reconstruct the holds on a climbing route after a brief preview (Boschker et al., 2002). Pezzulo et al. (2010) extended this to a realistic indoor climbing environment where expert climbers demonstrated better recall for climbing holds. Interestingly, this effect was only present in experts when the tested routes were difficult (in line with expert's ability level); there was no difference in recall ability between climbers of varying expertise when easy or impossible routes were tested (Pezzulo et al., 2010). This suggests that one's ability to actually climb a route may be related to their cognitive processing of visual information within a route.

The current study seeks to expand upon the findings of this enhanced visual memory effect in expert climbers. Expert climbers may be able to chunk visual information on a route into a manageable number of meaningful units, similar to chess experts (Chase \& Simon, 1973; De Groot, 1965). For climbers, a chunking mechanism may be aided by accurate perception of climbing moves via improved affordance perception (Boschker et al., 2002; Pezzulo et al., 2010), and/or motor simulation (Pezzulo et al., 2010). Both arguments assume that as rock climbers develop expertise, they develop the ability to more accurately assess and/or simulate what their body is capable of in relation to hold arrangements, which ultimately helps them chunk information on a route. If expert climbers can more effectively remember the holds on a route, due to body-based mechanisms, then they should also be able to more effectively plan and remember the movement sequences used to climb a route. Visual memory for holds on a route has been tested across levels of expertise (Boschker et al., 2002; Pezzulo et al., 2010); however, memory for movement sequences performed by climbers has yet to be tested. It is one task to identify what holds belong to a to-be-climbed route, but it is a more complex and dynamic task to plan how one's body will interact with those holds, in order to plan a sequence. Visual memory is important for climbers, but using that information to build a sequence of body movements is a critical final planning step in order to successfully climb. Given this, it can be inferred that more experienced climbers would be able to more successfully plan and remember the movement sequences for routes, yet this has not been experimentally evaluated.

We conducted two experiments to assess perceptual and cognitive expertise advantages of climbers. In Experiment 1, we examined climbers' perception of their ability to act upon target holds from a variety of starting positions on a climbing wall. In Experiment 2, we assessed climbers' abilities to predict and remember the motor sequences they would/did use on a climbing wall, and aimed to replicate the visual memory advantages previously found in expert climbers. We predicted that more skilled climbers would more accurately judge their ability to execute singular moves, and have better visual memory, sequence prediction, and sequence memory. Together, these studies aim to understand perceptual and cognitive skills that contribute to rock climbing expertise. 


\section{Experiment 1}

In Experiment 1, we tested perception of basic overhead reaching affordances within climbers of varying skill levels, and perception of climbing specific affordances. Experiment 1 was designed to extend previous work that has shown more accurate overhead reach judgments in climbers, compared with nonclimbers (Pijpers \& Bakker, 1993). Further, it was designed to assess climbing specific affordances in a realistic climbing environment, which may provide indirect support for the idea that accurate perception of climbing moves underlies the previous visual memory advantages found in expert climbers (Boschker et al., 2002; Pezzulo et al., 2010). Additionally, we sought to investigate if, alongside being more aware of their capabilities, more skilled climbers would be more aware of the effort needed for a given move.

\section{Hypotheses}

We had three hypotheses for Experiment 1: (1) more skilled climbers would show higher accuracy in determining whether they could perform single moves on a climbing wall, (2) more skilled climbers would be more accurate in judging their basic overhead reaching abilities, and (3) more skilled climbers would be able to more accurately predict how difficult a climb would be in relation to how difficult they judged it to be after climbing.

\section{Method}

Participants Thirty-four local climbers (14 males) between the ages of 18 and 34 years $(M=24.76, S D=5.54)$ were recruited from the Salt Lake City community. The V scale was used to measure climbing ability, which ranges from V0 to V17 (Sherman, Head, Crump, \& Head, 1991). Each value on the scale represents the difficulty level of boulder routes, which are distinctive preset hold arrangements on short climbing walls (typically less than 15-20 feet) that climbers ascend without a rope. V0 represents a difficulty level that most novices can climb, whereas V17 represents a difficulty level that only a handful of experts worldwide can climb. Participants self-reported skill levels ranged from V0 to V10 $(M=4.14$, $S D=2.64)$ and years of experience ranged from 0 to 20 years $(M=5.06, S D=4.23)$. Participants were recruited by experimenters, either via a psychology participant pool or by verbal advertising at the climbing gym. All participation was voluntary, and participants were given partial psychology course credit or US\$10. The experimental procedures and recruitment process were approved by an official local ethics committee.

Materials The experiment took place on preset climbing wall sections that varied in steepness at The Summit Climbing
Gym at The University of Utah's Campus Recreation Center. Each wall contained climbing holds varying in size, shape, and color to imitate rock features found in outdoor climbing environments. Six climbing hold sets were selected (three on a steep section of the wall, three on a vertical section of the wall) to serve as start holds (hands and feet). For each start hold set, at least one handhold and two footholds were chosen. Only two footholds were specified for each set because many of the target holds could have been easily reached if participants foot movements were not constrained, and consideration of foothold location is critical to planning and executing movements on a route. Three handholds in the vicinity of each start hold set were chosen as target holds. Each start hold set and the corresponding target holds were referred to as an array (see Fig. 1 for an illustrated example array). Each array consisted of holds that did not correspond to any existing route in the gym. This was done to reduce the possibility that participants would have prior exposure to the climbing movements for the tasks in this experiment. For the basic reaching task, a laser pointer was fixed to a camera tripod so that the experimenter could raise and lower a laser light projected onto a blank wall from 10 feet away.

Design We used a within-subjects design, where participants made judgments of their perceived ability to complete individual climbing moves (as well as how difficult they thought the climbing move would be to complete) in one block, and then physically attempted each move that was judged in another block (and then again rated the difficulty after they had actually tried the move). Participants completed all the judgments before making any attempts. A similar design was used to assess basic reaching ability, in which participants judged the height they perceived they could reach on the wall, and then performed actually reaching on the wall. Expertise was evaluated via a climbing experience questionnaire filled out by the participant at the end of the experiment.

Procedure All participants signed an informed consent document prior to beginning the experiment. Afterward, the experiment consisted of two blocks: a judgment block and a performance block. In the judgment block, participants were instructed to examine the start hold set of the first of six arrays (marked with tape, as well as illuminated by a laser pointer). Participants then judged whether they could get to and maintain grasp on each target hold within the array for a minimum of three seconds without using other holds on the wall. ${ }^{1}$ After they provided their yes or no judgment, participants were asked for a personal difficulty rating for the given move on a scale of 1 to 10 ( 1 representing no challenge and 10 representing maximal challenge). This procedure was

\footnotetext{
${ }^{1}$ We chose a 3 -second limit on hold time because this is a typical limit used in competitive climbing events.
} 


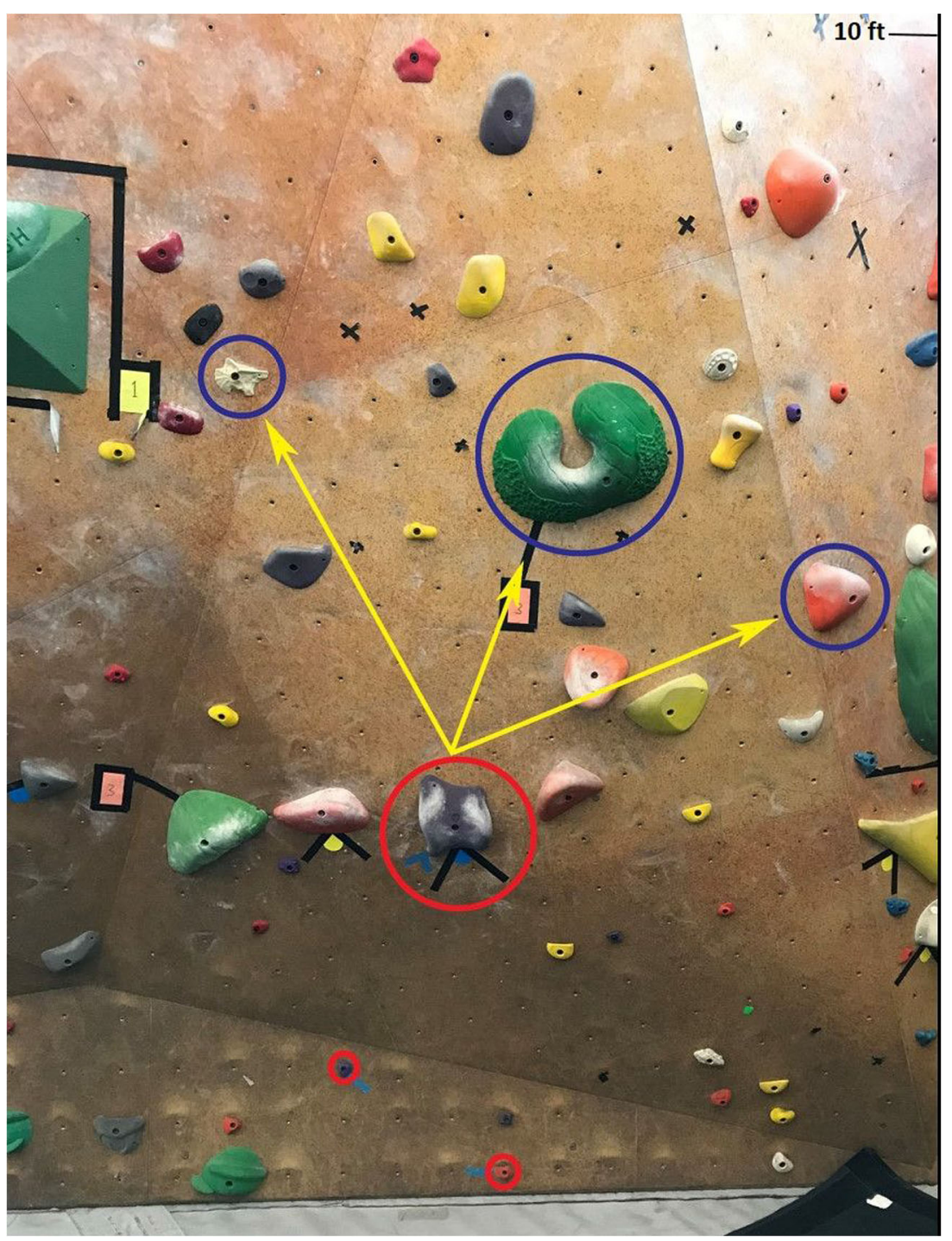

Fig. 1 Illustrated example array

repeated for each of the six arrays. Participants were randomly assigned to one of two array orders such that they made judgments of arrays in serial order (1-6), or in reverse order (6-1).

After the judgment block, participants were allowed up to 10 minutes to warm up before starting the performance block. After the warm-up, participants were encouraged to attempt each move in the judgment block. Successful and unsuccessful attempts were marked by the experimenter. A successful move was recorded when a participant was able to grasp a target hold and hold on for a minimum of 3 seconds. If the participant was confident that they could not complete the move, or if they did not want to try the move, they were allowed to skip the move and it was marked as an unsuccessful attempt. After the participant attempted each move, they were again asked for a personal difficulty rating. In all aspects of the performance block, participants were instructed to ignore previous judgments and only report on the current task.
This procedure was repeated for each of the six arrays in the same order as the judgment block.

Once participants had completed both the judgment and performance block, they completed a questionnaire to gather information about their demographics and climbing experience. Participants then performed a basic overhead reaching task. In this task, a laser pointer attached to a tripod was placed behind and to the side of the participant, so the experimenter could move the laser up or down a wall via the tripod, while the tripod remained out of the participant's field of view. Participants stood on a marked line 10 feet away from a blank wall and were instructed to tell the experimenter to stop when the laser dot height corresponded with what they judged to be their maximum reach height if they were standing against the wall, extending their arm upwards with heels on the ground. After the participant said "stop," they were able to instruct the experimenter to make adjustments to the height of the dot. Once the participant was satisfied with the laser dot height, 
they were asked to turn their back to the wall while the experimenter recorded the height. Each participant judged their reaching height two times: one trial where the dot moved downward from the ceiling, and one trial where it moved upward from the floor, trial order was counterbalanced per participant. Once both trials were completed, participants stepped to the wall with their hand above their head and their actual reach height was recorded. Finally, participants were debriefed and compensated for their participation.

\section{Results}

Data analysis For our three main hypotheses we conducted separate regression analyses to test the effect of skill level on perceptual judgments, reach judgments, and difficulty ratings. We did not conduct a priori sample size estimates; however, our post hoc sensitivity analysis revealed that the smallest reliable effect size in standardized regression units we could reliable detect was 0.44 given our sample size and an assumed power of $0.8 .^{2}$ All of our main findings met this threshold, and one of our exploratory findings did not; we discuss the implications of this in our Limitations section. To determine skill level, responses to two questions on the questionnaire were averaged into a single value: hardest bouldering route completed outdoors, and hardest route completed indoors (both on a V scale). ${ }^{3}$ This value was labeled as the skill level and was used as the indicator of expertise throughout the analyses. ${ }^{4}$ Perceptual judgment accuracy scores were created by taking the proportion of accurate responses divided by overall responses. Judgments were categorized as accurate when participants said they could complete a move in the judgment block and were able to do so in the performance block, or judged they could not complete a move in the judgment block and could not in the performance block. For the basic reaching task, a reach ratio was calculated in which participants' average estimated overhead reach was divided by their actual overhead reach. For difficulty ratings, a difference score was created between pretest and posttest difficulty ratings. Difficulty-difference scores for all 18 trials were averaged to calculate a mean difficulty change rating for each participant,

\footnotetext{
${ }^{2}$ Post hoc sensitivity analysis was conducted in $\mathrm{G}^{*}$ Power, Version 3.1.9.2 (Faul, Erdfelder, Lang, \& Buchner, 2007). Alpha was set to 0.05, power was set to 0.8 , sample size was set to 34 , and we specified that our test was twotailed. The slope for the null hypothesis was set to zero and the standard deviations for $x$ and $y$ were set to the default value of one.

${ }^{3}$ We used an averaged value to try to encompass overall climbing ability (both indoors and outdoors). However, we could have reasonably used either indoor skill value (hardest route completed indoors on a V scale) or the indoor/ outdoor averaged value as they were highly correlated, $R=.97$.

${ }^{4}$ The skill level value used for the experiment was correlated with years of climbing experience, $R=.39$. All results were replicated with years of experience in the same direction and with similar magnitude. We chose to use

skill level as our proxy for expertise instead of years of experience because of potential variation in climbing frequency over the years as well as type of climbing (bouldering, route climbing, traditional climbing, etc.).
}

Table 1 Descriptive statistics for Experiment 1

\begin{tabular}{lc}
\hline Variable & $M(S D)$ \\
\hline Perceptual judgment accuracy & $.7(.13)$ \\
Reach ratio & $1.01(.06)$ \\
Preclimb difficulty & $6.26(2.79)$ \\
Postclimb difficulty & $5.88(3.27)$ \\
Difficulty-difference score & $2.06(.59)$ \\
Overconfident error proportion & $.22(.17)$ \\
Underconfident error proportion & $.08(.11)$ \\
\hline
\end{tabular}

Note. Standard deviations are in parentheses

thereby allowing us to compare abilities in predicting the difficulty as a function of climbing expertise.

We ran two exploratory analyses, after we noticed a pattern in the types of errors that climbers of varying skill level made in their yes/no ratings of whether they could complete the movement in their judgment block (as compared with their observed ability in the performance block). Responses were categorized into underconfident and overconfident errors. Errors were categorized as overconfident when participants said they could do a move in the judgment block but were unable to complete the move in the performance block. Errors were categorized as underconfident when participants said they could not do a move in the judgment block but were able to complete the move in the performance block. As with our main analysis of skill level on perceptual judgment accuracy, errors were converted into a proportion of the total number of possible responses (Table 1).

Main findings: Accuracy, reach ratio, and difficulty score Three linear regressions were conducted with regard to our stated hypotheses. First, a linear regression was conducted to determine the relationship between skill level and accuracy, in which participants' accuracy was regressed onto their skill level. Skill level was significantly related to accuracy ( $B=$ $.028, \beta=.544, p<.001)$, such that a one-unit increase in skill level would predict a $2.8 \%$ increase in participants' proportion of accurate responses (see Fig. 2). Next, a linear regression was conducted to determine the relationship between skill level and reach ratio, in which reach ratio was regressed onto skill level. Skill level was not significantly related to reach ratio $(p=.99)$. Finally, a linear regression was conducted to determine the relationship between skill level and average difficulty-difference score (pre - post), in which average difficulty-difference score was regressed onto skill level. Skill level was not significantly related to average difficultydifference score $(p=.3)^{5}$.

\footnotetext{
5 Although skill level was not related to participants' difficulty-difference scores, we investigated if participants' pretest difficulty ratings were related to their actual performance with a mixed logistic regression model. The model showed that higher pretest difficulty ratings were indeed associated with higher odds of a failure $(B=0.149, p<.001)$.
} 


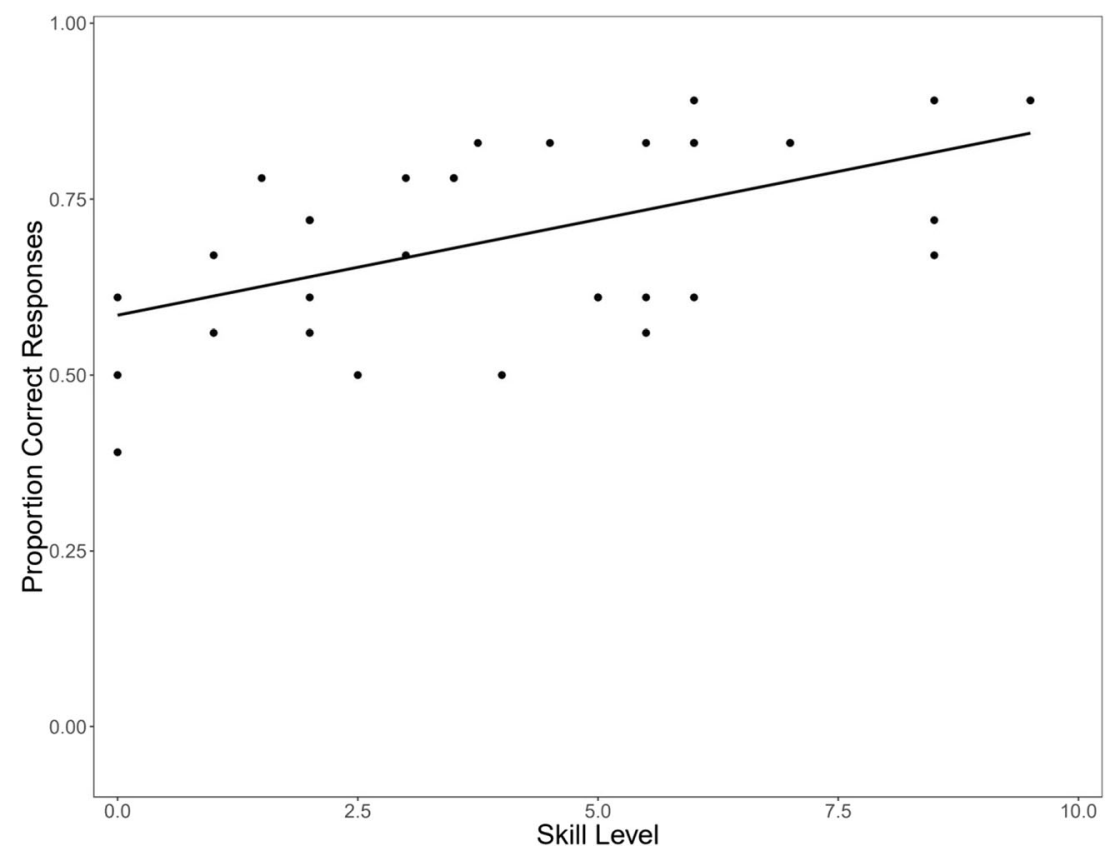

Fig. 2 Scatterplot of correct responses as defined by accurately predicting whether the move could be completed in the judgment block when compared with success in the performance block, with regression line overlaid

\section{Exploratory findings: Underconfident and overconfident er-} rors Linear regressions were conducted in which participants' proportion of underconfident or overconfident errors were regressed onto their skill level to explore post hoc error patterns. We found that skill level was significantly related to both underconfident $(B=.014, \beta=.350, p=.042)$, and overconfident $(B=-.0412, \beta=-.654, p<.001)$ errors, albeit in opposite directions, such that a one-unit increase in skill level would predict a $1.4 \%$ increase in underconfident errors, and a $4.1 \%$ decrease in overconfident errors (see Fig. 3).

\section{Discussion}

In Experiment 1, we had three hypotheses related to perception of action capabilities in climbers of varying skill levels. First, we predicted that more skilled climbers would show higher accuracy in determining whether or not they could do single moves on a climbing wall. Our results supported this hypothesis, revealing that as skill increased, perceptual judgment accuracy increased. Next, we predicted that more skilled climbers would more accurately judge their basic maximum overhead reach capabilities. We did not find evidence to support this prediction, as there was not a significant relationship between reach ratio and skill level. Previous work has shown that experienced climbers are more accurate on a basic overhead reaching task compared with nonclimbers (Pijpers \& Bakker, 1993). Our results suggest that such differences may not be present when comparing within climbers of varying skill levels. However, the climbers in our study were near perfect with their reach estimates, and there was little variation so our null result may have also been due to ceiling effects.
Finally, we predicted that more skilled climbers would more accurately predict how difficult the route would be, indicated by greater similarity between the predicted difficulty in the judgment block and the rated difficulty in the performance block. We did not find evidence to support this prediction, as there was not a significant relationship between average difficulty score change and skill level. It is possible that predicting difficulty in more abstract numeric terms is less critical to climbing success than being able to predict what one's body is capable of. Additionally, we found an unexpected result when we examined the types of errors that climbers of varying skill level made. More skilled climbers made more underconfident errors (i.e., underestimating their abilities), whereas less skilled climbers made more overconfident errors (i.e., overestimating their abilities) in their perceptual judgments. Overall, these results suggest that skill level influences perceptual accuracy, and possibly the types of errors climbers make when they assess action capabilities in a climbing environment.

\section{Experiment 2}

In our first experiment, we found evidence to suggest a climber's skill level is positively associated with their ability to perceive single moves on a climbing wall. This result potentially provides indirect support for the idea that climbers' perception of action capabilities underlies previously found visual memory advantages (Boschker et al., 2002; Pezzulo et al., 2010). However, the perception of single climbing moves may not fully explain visual memory of full routes, 


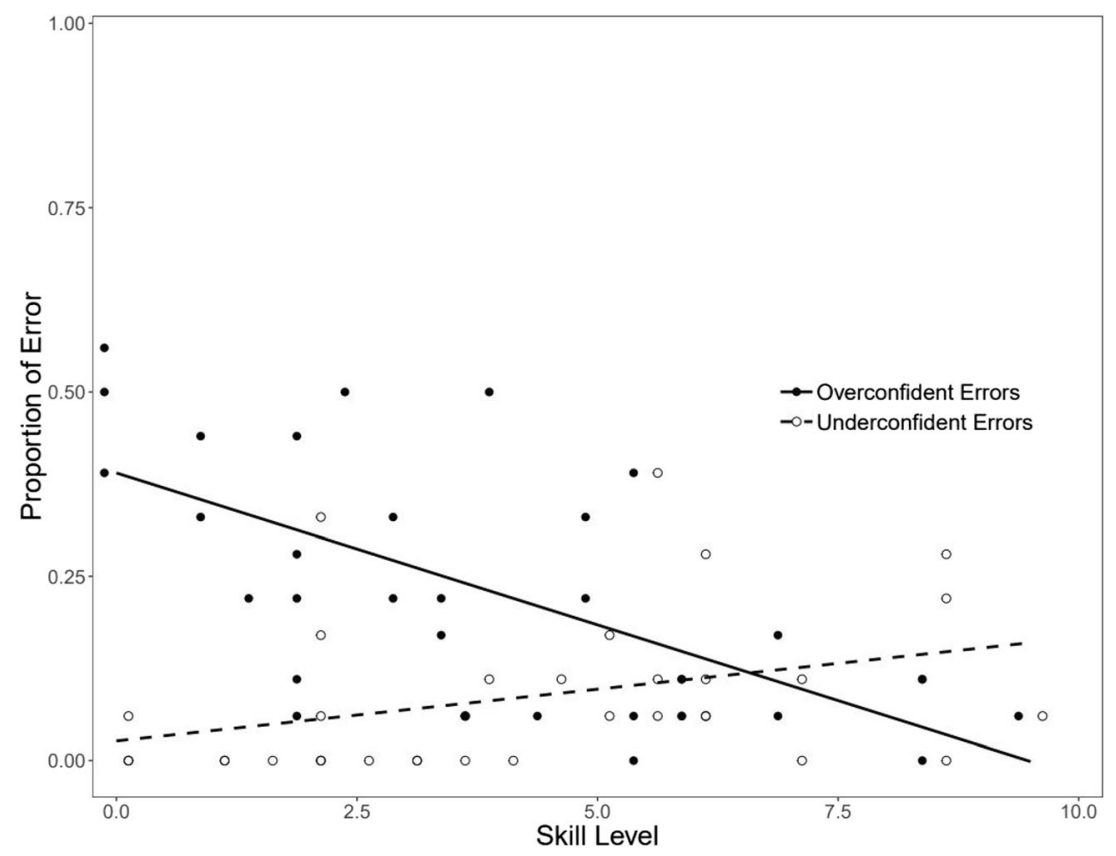

Fig. 3 Scatterplot of underconfident (responded they could not complete the move in the judgment block, but successfully completed it in the performance block) and overconfident errors (responded they could

complete the move in the judgment block, but did not complete it in the performance block), with regression lines overlaid

because a complete route consists of a multiple-move sequence. Our goal in Experiment 2 was to test if a climber's skill level was related to their ability to plan motor sequences and remember performed actions on a climbing wall (sequence memory). Furthermore, we sought to replicate previous visual memory advantages seen in expert rock climbers (Boschker et al., 2002; Pezzulo et al., 2010). Additionally, we wanted to account for general visuospatial working memory, to assess if superior sequence memory in skilled climbers would be a domain-specific or domain-general skill. There is mixed evidence as to whether general visuospatial working memory performance is higher in movement experts. Barhorst-Cates (2018), showed that dancers outperformed controls on both a traditional and embodied Corsi task, measuring visuospatial working memory. However, there has also been work showing that basketball players and gymnasts do not outperform controls on a traditional Corsi task (Furley \& Memert, 2010; Pereira, Abreu, \& Castro-Caldas, 2013).

\section{Hypotheses}

We had three hypotheses for Experiment 2: (1) more skilled rock climbers would have better visual memory of holds on the climbing wall compared with less skilled rock climbers, both after a 1-minute preview and after interacting with the holds while attempting to climb the route; (2) more skilled rock climbers would more accurately predict the sequence of movements they would use to execute moves on a climbing route after a 1-minute preview of the route compared with less skilled climbers; and (3) more skilled rock climbers would remember climbed sequences for rock climbing routes more accurately compared with less skilled climbers. Additionally, we evaluated general visuospatial working memory ability as it relates to climbing expertise as an exploratory variable.

\section{Method}

Participants Twenty climbers (eight females) between the ages of 20 and 45 years $(M=27.55, S D=6.50)$ were recruited from a local indoor climbing gym. Self-reported skill levels ranged from V3 to V13 $(M=7.1, S D=2.43)$, and years of experience from 0.5 to 25 years $(M=7.65, S D=6.87)$. All participation was voluntary, and participants were compensated US $\$ 10$ for their participation. The experimental procedures and recruitment process were approved by an official local ethics committee.

Materials The experiment took place at The Front Climbing Club in Salt Lake City, Utah. A bouldering wall section (16 ft) that had been cleared and reset was selected for the experiment, to ensure that participants had no prior experience with the routes. The holds that belonged to each route were distinguished by the color of the holds along that route (both hands and feet). A composite image was compiled to capture all wall sections used for memory testing. That compiled image was then converted into a modified grayscale image that removed color, so participants could not easily identify holds belonging to a particular route. A grayscale image was used because each boulder route had a unique color and the task would have been too easy if a color image was used for memory testing. This 
grayscale image was used for the visual and sequence memory tests. Participants were presented with the image on a tablet (Microsoft Surface Pro or iPad) and indicated their responses to the hold location and climbing sequence using a stylus pen.

To assess general visuospatial working memory, participants completed the Corsi block-tapping task (Corsi, 1972), adapted by our lab for computer presentation in Unity. During the Corsi block-tapping task, an arrangement of blocks was displayed on the screen. During each trial, a single block would illuminate at a time indicating the to-be-remembered sequence. After the sequence was complete on each trial, a prompt appeared on the screen instructing the participant to repeat the sequence by clicking on the blocks in the order in which they had illuminated. Sequence length ranged from two to nine. All participants completed the Corsi block-tapping task on a Dell laptop.

Design We used a within-subjects design, where participants each completed visual and sequence memory tasks before and after they climbed three routes of their choice. Participants completed each of these tasks one route at a time. All participants also completed the Corsi block-tapping task and a questionnaire that assessed their climbing experience and expertise.

Procedure Participants were informed of an experiment taking place via verbal introduction by experimenters at the climbing gym. Interested climbers first agreed to and signed an informed consent document. Next, participants completed a questionnaire to gather information about their demographics and climbing experience. Depending on the laptop availability, participants either completed the Corsi block-tapping task after completing the questionnaire, or after completing the visual and sequence memory tasks.

For the visual and sequence memory tasks, each participant was first instructed by the experimenter to view the newly set routes and select three out of the 12 climbs: one climb that they thought they could definitely do, one they thought they could likely do but would be challenging, and one they thought would be difficult or out of their ability. Participants were tested on the three selected routes for both the visual and sequence memory tasks. Because our participants varied in terms of skill level, this selection technique was used as an attempt to match the climbers' effort level, to avoid having skilled climbers perform only very easy climbs or beginner climbers only attempt routes they were incapable of doing. All routes were newly set, and to further ensure all routes were novel, participants were instructed to only select routes that they had not yet attempted. After participants had selected three routes, they were allotted a 1-minute preview to inspect and plan a sequence on each route. During the preview, participants were permitted to move freely to inspect the routes, but were prohibited from touching the wall or any of the holds.
Once they felt confident in their sequence, or the 1-minute preview time was up, they turned their backs to the climbing wall to prevent further visual inspection. Participants then viewed the grayscale image on the tablet, and were instructed to complete the visual memory pretest by indicating all hand holds on the display that belonged to the route they had just previewed by putting a dashed line through the center of the hold with the stylus pen. Next, participants completed the sequence prediction task. Participants were instructed, with view of the holds they had selected during the visual memory task, to first indicate their predicted hand placement for the start of the route by writing " $\mathrm{R}$ " for right and " $\mathrm{L}$ " for left next to the starting hold/s. Participants also labeled the order of each hand movement and which hands would make each movement for the entire route. For example, if they planned to make their first move with their right hand, they would mark that hold "R1," and if they planned to make their next move with their left hand, they would mark that hold "L2." If at any time they planned to use both hands on a hold, they would mark that hold with both " $R$ " and " $L$ " together with the corresponding move number. We did not ask them to mark footholds, in order to avoid excessive challenge on the sequence memory task (see Fig. 4 for an example participant visual memory pretest and sequence prediction response). An experimenter thoroughly explained both the visual and sequence memory tasks to the participant before starting any trial. During all memory tests, participants were able to mark the tablet and make any changes or edits, and were instructed to hand the tablet back to the experimenter when they were finished. Upon completion of the visual memory pretest and sequence prediction tasks, participants' sequences were recorded as they attempted to climb their chosen route up to five times, taking as much time to rest in between attempts as desired. After they had successfully ascended the route, used their five attempts, or decided to stop making attempts, participants once again turned their backs to the wall and completed the memory tests on the tablet. The postclimb visual memory test was identical in instruction to the visual memory pretest. For the postclimb sequence memory test, participants indicated the sequence they remembered climbing (not the sequence they predicted, if different) using the same procedure as the sequence prediction task. Participants then moved on to the second and third routes, repeating this procedure for each. Finally, participants were debriefed and given compensation for their participation.

\section{Results}

Data analysis Prior to data analysis, we excluded trials where participants used four or fewer holds when attempting a route. We did this in order to make sure that we did not inflate participants' performance if they only completed a small portion of the route and thus only needed to remember 1-4 moves 


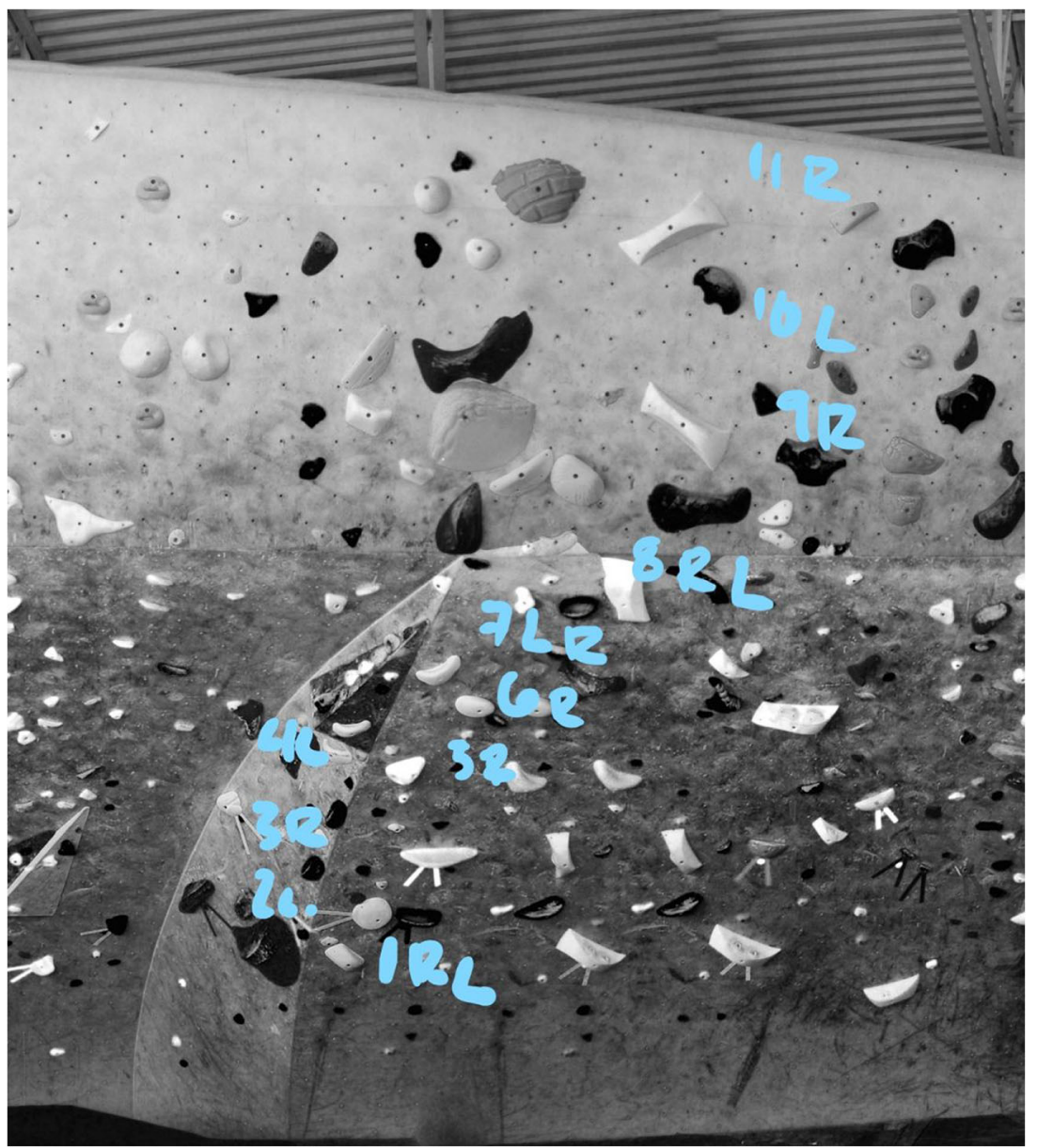

Fig. 4 Sample participant response to the visual and sequence memory tasks

(i.e., in the case of sequence memory). However, by doing so, we created an unbalanced data set where some participants had less than six trials worth of data (maximum of three pretest and three posttest trials). Therefore, we chose to use a multilevel modeling (MLM) approach, which is a form of generalized regression that can partition out variance within and between individuals. Importantly, MLM provides an appropriate means to analyze unbalanced data and nested experimental designs (Raudenbush \& Bryk, 2002).

Visual analysis of histograms and QQ plots of our outcome variables indicated nonnormal distributions that resembled a Poisson form. Poisson distributions are highly skewed toward zero, have long positive tails, and are appropriate for count type data such as number of errors. Therefore, we ran our MLM models under the assumption of a Poisson distribution, which has a log link function and represents the outcome variables as an event rate. In our case, the events indicate the amount of visual/sequence errors expected per trial. Hierarchical linear modeling (HLM 7.03; Raudenbush, Bryk, \& Congdon, 2011) and restricted maximum likelihood estimation was used for all our models.

In total, we report three Poisson MLM analyses where we investigated the effects of skill level on visual and sequence memory. We analyzed each test of sequence memory (preclimb and postclimb) in separate models because the errors in each case were of a different nature. Prior to climbing, sequence memory errors reflected errors in the climbers' ability to predict what moves they would make to climb the route. Errors made postclimb indicated errors in the climbers' ability to remember the climbing moves they used. Post hoc sensitivity analyses were conducted for the visual memory model and sequence models separately, and used the same inputs specified in Experiment 1. Sample sizes were estimated for each sensitivity analysis using the $\mathrm{N}$-effective formula (Kenny, Kashy, \& Cook, 2006), which accounts differences in the intraclass correlation coefficient, the number of groups sampled (in our case, individuals), and the number of repeated measures observed within each group. Our sensitivity analyses revealed that the minimal detectable effect sizes (in standardized beta units) for our visual memory and sequence models were 0.50 and 0.51 , respectively. All effects of skill level were above these thresholds, except for the effect of skill level on sequence errors preclimb, which was 0.005 standardized units lower than the cutoff. Given that this difference is extremely small, we are confident that are results 
are reliable at our assumed power level of 0.8. The three MLM equations are shown below in series of equation format:

\section{Visual Memory;}

Level 1

$$
\begin{aligned}
& E\left(\operatorname{VISERROR}_{i j} \mid \beta_{j}\right)=\lambda_{i j} \\
& \log [\lambda i j]=\eta_{i j} \\
& \eta_{i j}=\beta_{0 j}+\beta_{1 j} \times\left(\text { TEST }_{i j}\right)
\end{aligned}
$$

Level 2

$$
\begin{aligned}
& \beta_{0 j}=\gamma_{00}+\gamma_{01} \times\left(\text { SKILL_GC } C_{j}\right)+u_{0 j} \\
& \beta_{1 j}=\gamma_{10}+\gamma_{11} \times\left(\text { SKILL_GC } C_{j}\right)+u_{1 j}
\end{aligned}
$$

\section{Sequence Memory Before Climb;}

Level 1

$$
\begin{aligned}
& E\left(\operatorname{SEQERROR} R_{i j} \mid \beta_{j}\right)=\lambda_{i j} \\
& \log [\lambda i j]=\eta_{i j} \\
& \eta_{i j}=\beta_{0 j}
\end{aligned}
$$

Level 2

$$
\beta_{0 j}=\gamma_{00}+\gamma_{01} \times\left(S K I L L_{-} G C_{j}\right)+u_{0 j}
$$

\section{Sequence Memory After Climb;}

\section{Level 1}

$$
\begin{aligned}
& E\left(\operatorname{SEQERROR} R_{i j} \mid \beta_{j}\right)=\lambda_{i j} \\
& \log [\lambda i j]=\eta_{i j} \\
& \eta_{i j}=\beta_{0 j}
\end{aligned}
$$

\section{Level 2}

$$
\beta_{0 j}=\gamma_{00}+\gamma_{01} \times\left(S K I L L_{-} G C_{j}\right)+u_{0 j}
$$

where $i$ represents trial (i.e., a single observation) and $j$ represents individuals. Level 1 represented the variability in the repeated trials within participants, and Level 2 represented the variability between our participants' skill level. Test type was included as a dummy coded predictor $(0=$ preclimb, 1 = postclimb) at Level 1 in the visual memory model. Grand centered skill level, which is a given individual's skill level subtracted from the overall mean skill level of the sample, was added as a predictor at Level 2 for all models. Importantly, we did not include participants' visuospatial working memory as a predictor in any of the models above because preliminary analyses revealed no significant relationship between participants' skill level and Corsi scores $(\beta=.006, p=.84){ }^{6}$

Expertise For expertise, responses to two questions on the questionnaire were averaged into a single value: hardest bouldering route completed outdoors (on a V scale), and hardest route completed indoors, indicated by dot color corresponding to $\mathrm{V}$ grade ranges at the Front Climbing Club. ${ }^{7}$ The color responses were converted to the numeric value at the center of that range before they were averaged together with the outdoor $\mathrm{V}$ scale value. This value was labeled as the skill value and was used as the indicator of expertise throughout the analyses. ${ }^{8}$

Visual memory Table 2 shows the results of our analysis of climbing skill on visual memory of boulder route holds. The model revealed a negative association between visual memory errors and climbing skill, $\left(\gamma_{01}=-.20, S E=.053, p=.002\right)$. The coefficients in these models are in log event rate form and to make them more interpretable we transformed the coefficients into a proportion of errors with the following formula: $1-\mathrm{e}^{[(\gamma)}$ (x)] , where $\gamma$ represents the log event rate coefficient and $x$ represents a value for the corresponding predictor (e.g., a value of skill level; Raudenbush \& Bryk, 2002). Therefore, the aforementioned effect indicates that that a one standard deviation increase in climbing skill $\left(S D_{\text {climbskill }}=2.87\right)$ is predicted to decrease the visual error event rate by $1-\mathrm{e}^{[(-0.20)(2.87)]}$ $=.44$ or $44 \%$. There was also a strong negative association between visual memory errors and test (preclimb and postclimb), $\left(\gamma_{10}=-.824, S E=.111, p<.001\right)$. After the participants climbed a boulder route, their predicted visual memory error event rate would decrease by $56 \%$ for each boulder route. Importantly, there was no significant cross-level interaction between test and climbing skill $\left(\gamma_{11}\right)$, which indicates that the effects of climbing a boulder route on visual memory did not depend on the climbers' skill level. In other words, all climbers experienced similar improvements in visual memory after they climbed the route. We supplement these results with Fig. 5, which depicts the predicted number of visual memory errors per boulder route based on the model in Table 2 .

Sequence error preclimb Table 3 depicts the results of the preclimb sequence error model, which revealed a significant

\footnotetext{
$\overline{6}$ There was also no significant relationship between participants' Corsi scores and error on the visual and sequence tasks.

${ }^{7}$ We used an averaged value to encompass overall climbing ability (both indoors and outdoors). However, we could have reasonably used either indoor skill value (hardest route completed indoors on a V scale) or the indoor/ outdoor averaged value as they were highly correlated, $R=.96$.

${ }^{8}$ The skill level value we used for the experiment strongly correlated with years of climbing experience, $R=.79$. We chose to use skill level as our proxy for expertise instead of years of experience due to potential variation in climbing frequency over the years as well as type of climbing (bouldering, route climbing, traditional climbing, etc.).
} 
Table 2 Model results of visual memory error

\begin{tabular}{lrrrrr}
\hline Parameter & Coefficient & \multicolumn{1}{l}{$S E$} & \multicolumn{1}{l}{$t$} & Approx. $d f$ & $p$ \\
\hline$\beta_{0}$ & & & & & \\
Intercept, $\gamma_{00}$ & 0.33 & 0.145 & 2.261 & 18 & .036 \\
Skill, $\gamma_{01}$ & -0.20 & 0.053 & -3.656 & 18 & .002 \\
Test, $\beta_{1}$ & & & & & \\
Intercept, $\gamma_{10}$ & -0.82 & 0.111 & -7.404 & 18 & $<.001$ \\
Skill, $\gamma_{11}$ & -0.04 & 0.052 & -0.717 & 18 & .483 \\
\hline
\end{tabular}

Note. Results present the population average coefficients in log event rate form

negative association between preclimb sequence memory error and climbing skill, $\left(\gamma_{01}=-.21, S E=.056, p=.002\right)$. Specifically, the model predicted that a one standard deviation increase in climbing skill $\left(S D_{\text {climbskill }}=2.87\right)$ would decrease climbers' preclimb sequence memory error event rate by $45 \%$ for each boulder route. In other words, climbers with higher skill level than their peers would be expected to make less errors if asked to predict their intended move sequence for a boulder route. Figure 6 shows the predicted number of preclimb sequence memory errors based on the model in Table 3.

Sequence error postclimb The results of our third model are shown in Table 4 . There was a significant negative association between postclimb sequence memory error and climbing skill, $\left(\gamma_{01}=-.26, S E=.044, p<.001\right)$. This indicates that a one standard deviation increase in climbing skill $\left(S D_{\text {climbskill }}=\right.$ 2.87 ) is predicted to decrease climbers' postclimb sequence memory error event rate by $53 \%$ per boulder route. Broadly,
Table 3 Model results of preclimb sequence error

\begin{tabular}{lrlrrr}
\hline Parameter & Coefficient & $S E$ & $t$ & Approx. $d f$ & $p$ \\
\hline$\beta_{0}$ & & & & & \\
Intercept, $\gamma_{00}$ & 0.84 & 0.145 & 5.756 & 18 & $<.001$ \\
Skill, $\gamma_{01}$ & -0.21 & 0.056 & -3.678 & 18 & .002 \\
\hline
\end{tabular}

Note. Results present the population average coefficients in log event rate form

this means that more skilled climbers make fewer errors if asked to report on the moves they just performed on a boulder route. Figure 7 shows the predicted number of postclimb sequence memory errors across the range of climbing skill based off the model results in Table 4.

\section{Discussion}

The current study tested three hypotheses aimed at better understanding factors that contribute to expertise in rock climbing. First, we predicted that more skilled rock climbers would have better visual memory for climbing holds than less skilled climbers, replicating previous work in the field (Boschker et al., 2002). Our results supported this hypothesis by revealing that the predicted rate of visual memory errors decreased as skill level increased both for the visual memory pretest and posttest. Next, we predicted that more skilled climbers would be better able to predict their intended climbing sequence after a 1-minute preview, and recall the sequence they used to climb the route compared with less skilled climbers. Both Hypotheses 2 and 3 were supported: sequence prediction error rates and sequence memory error

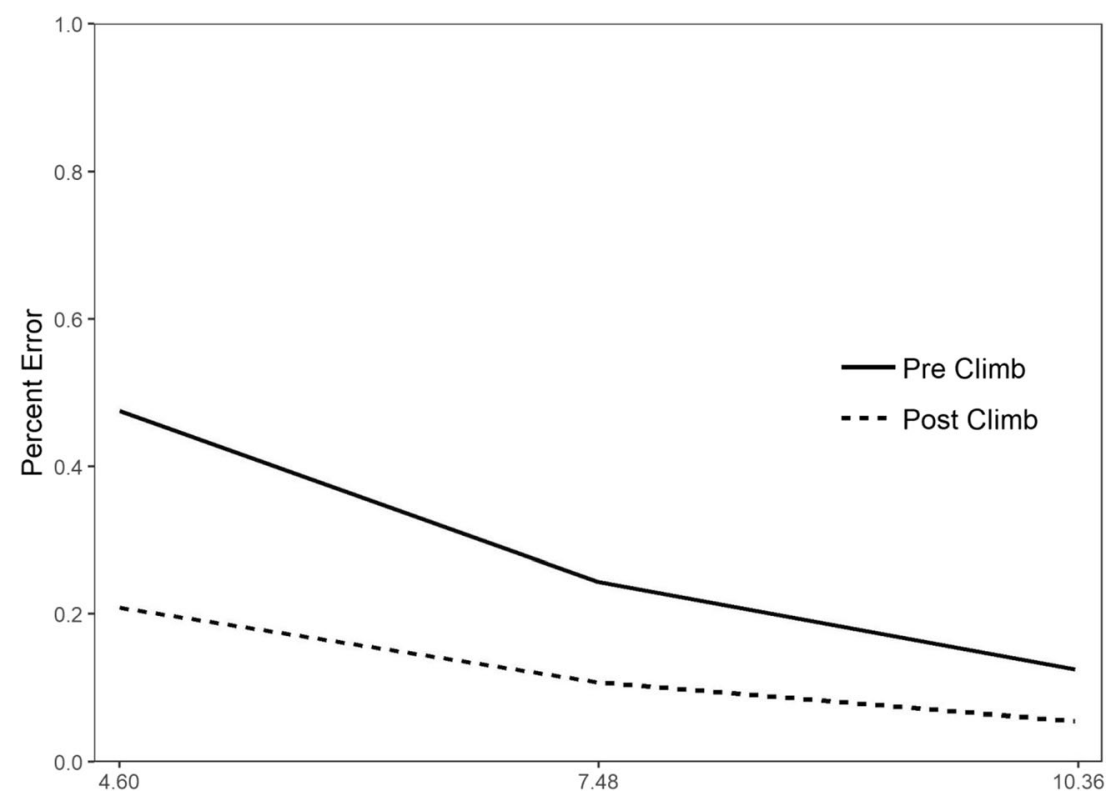

Fig. 5 A graph of the predicted visual memory error rate for mean and $\pm 1 S D$ climbing skill before and after climbing. Values represent the predicted rate (or percentage) of errors for a single visual memory trial as a function of skill level 


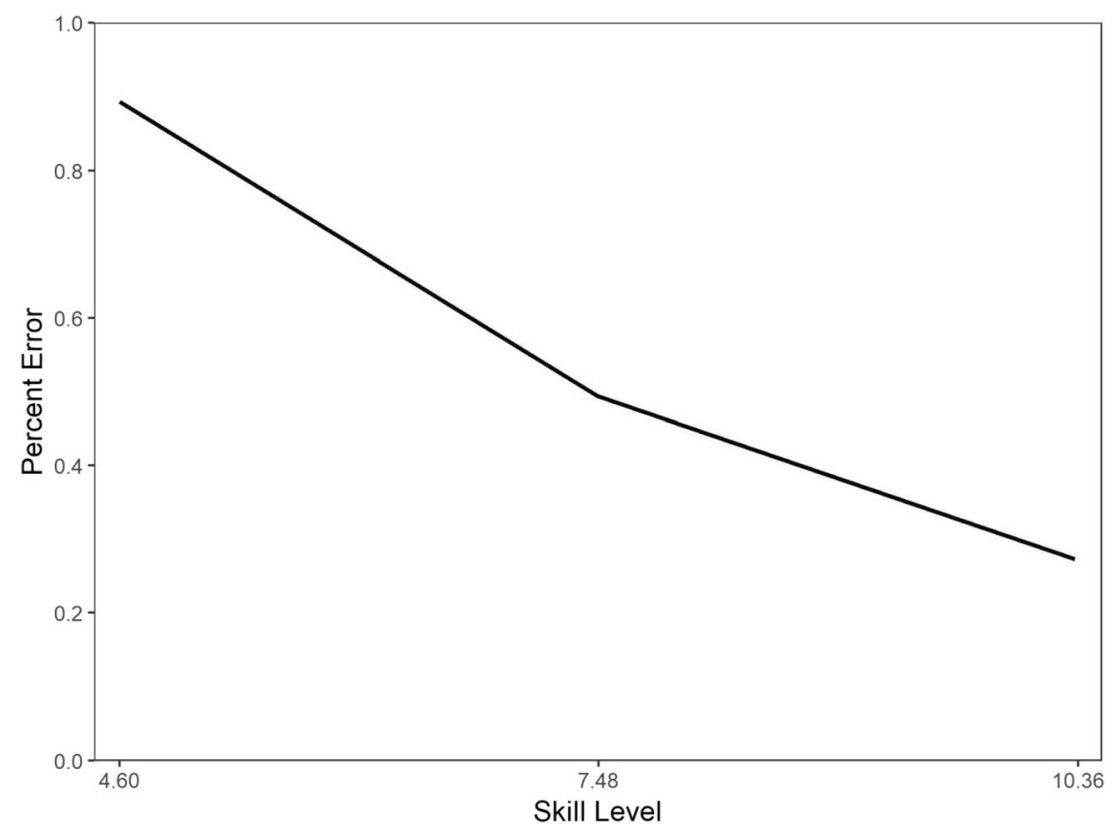

Fig. 6 The predicted sequence prediction error rate for mean and $\pm 1 S D$ climbing skill before climbing. Values represent the rate (or percentage) of error for a single boulder route trial as a function of skill level

rates decreased as skill level increased. Together, these findings provide support for the idea that knowledge of movement sequences used while rock climbing is an important cognitive skill to consider in climbing expertise. Finally, more skilled climbers did not outperform less skilled climbers on a domaingeneral assessment of visuospatial working memory, suggesting that enhanced sequence memory abilities in rock climbers are only evident for climbing-specific and not domain-general sequence tasks. The lack of association is consistent with findings from gymnast and basketball players, suggesting no influence of movement expertise on general sequence memory tasks (Furley \& Memert, 2010; Pereira et al., 2013). Yet it is inconsistent with other findings suggesting there is a benefit of movement expertise on general, as well as embodied, sequence memory tasks (Barhorst-Cates, 2018). Given conflicting research on whether or not domain-specific skills transfer to domain-general skills (Voyer \& Jansen, 2017), further investigation of this association is needed to claim whether or not domain-specific sequence ability in climbers generalizes to domain-general sequence ability.

Table 4 Model results of postclimb sequence error

\begin{tabular}{lrlrrr}
\hline Fixed effect & Coefficient & \multicolumn{1}{l}{$S E$} & \multicolumn{1}{l}{$t$} & Approx. $d f$ & $p$ \\
\hline$\beta_{0}$ & & & & & \\
Intercept, $\gamma_{00}$ & 0.27 & 0.190 & 1.441 & 18 & .167 \\
Skill, $\gamma_{01}$ & -0.26 & 0.044 & -6.072 & 18 & $<.001$ \\
\hline
\end{tabular}

Note. Results present the population average coefficients in log event rate form
Experiment 2 replicated previous work that shows a visual memory advantage for expert rock climbers (Boschker et al., 2002), extending the findings to another country, set of climbers, and climbing gym environment. Therefore, the visual memory advantage found in skilled rock climbers seems to be a robust effect that is likely not due to environmental factors (e.g., route settings, rating scales, social influences). Another unique component of our study is that we tested climbers' visual memory of routes before and after climbing a boulder route. Overall, these results suggest that expertise enhances climbers' cognitive abilities relevant to indoor rock climbing.

\section{General discussion}

Our results from two experiments provide evidence for both perceptual and cognitive expertise advantages of rock climbers. In Experiment 1, we found that more skilled rock climbers perceive their own action capabilities more accurately compared with less skilled climbers. In Experiment 2, we replicated the visual memory advantages previously found in expert rock climbers (Boschker et al., 2002), and extended climbing expertise effects to motor sequence memory. Our findings taken together reinforce that more skilled climbers possess cognitive and perceptual advantages. Specifically, they can sequence and remember large amounts of information and make accurate perceptual judgments of action capabilities pertinent to their domain.

In our first study, we found evidence for a previously unsupported claim used frequently in climbing expertise 
literature (Boschker et al., 2002; Pezzulo et al., 2010)-that expert climbers are more accurate at perceiving what their body is capable of, which may underlie higher level cognitive abilities like memory. Additionally, skilled climbers may be more likely to make underconfident errors, whereas less skilled climbers may be more likely to make overconfident errors. Less skilled climbers may attend to less relevant aspects of holds (e.g., shape and color) while expert climbers attend to more relevant aspects (i.e., how holds can be grasped; Boschker et al., 2002). More skilled climbers may have acquired a negative association for overconfident judgments because of undesirable outcomes (such as falls) resulting from overconfident judgments in their past climbing experience. Therefore, more skilled climbers may be more conservative in their judgments, in order to manage anxiety related to fear of falling, which has been shown to impair climbing efficiency (Nieuwenhuys, Pijpers, Oudejans, \& Bakker, 2008). Further, experts may be more tuned to the task demands of their environment, as a STOM (strategic task overload model) predicted time on task for expert, but not nonexpert, climbers (Wickens, McLaughlin, Keller, \& Tan, 2018). In being more aware of the task demands, experts may also be more aware of their shortcomings, leading them to underestimate their abilities. This assumption is in line with the unskilled-unaware effect in which low ability on social and cognitive tasks (i.e., humor assessment, logical reasoning, grammar knowledge) is related to overconfidence and high ability is related to underconfidence (Kruger \& Dunning, 1999). Our findings provide evidence that skill level influences accuracy - and may influence the types of errors that climbers make on a perceptual judgment task, yet the latter effects require more examination to make any strong claims and to disentangle potential mechanisms.

In our second study, we extended the literature on rock climbers' motor expertise to include sequence prediction and sequence memory, which are important cognitive tasks for successful climbing. Given that more skilled climbers did not outperform less skilled climbers on the Corsi blocktapping task, enhanced sequence planning and memory performance is likely not due to superior general visuospatial working memory, but to domain-specific ability. Additionally, given that visual memory was enhanced in experienced climbers, climbers may be either automatically or intentionally taking into consideration how their bodies could act on the holds, therefore enhancing their memory for the visual arrays. If this were the case, it would follow that more skilled climbers would be able to indicate and/or remember the body-based movements if directly tested. Indeed, we found that more skilled climbers are better able to predict and remember what sequence worked best for their bodies. Both of these findings support the assumption that skilled climbers use knowledge of what their bodies can or have done to aid with memory for rock-climbing routes.

Our studies were unique in that we examined perceptual and cognitive skills across a range of skill levels. Both our studies consisted of rock climbers with a wider range of skill than had been previously examined. Specifically, our sample included more intermediate climbers. The inclusion of this skill level range provides evidence of continuous progression of the perceptual and cognitive skills that we discuss. Further, our findings add to expertise literature showing more accurate perceptual judgments (Correia et al., 2012; Farrow \&

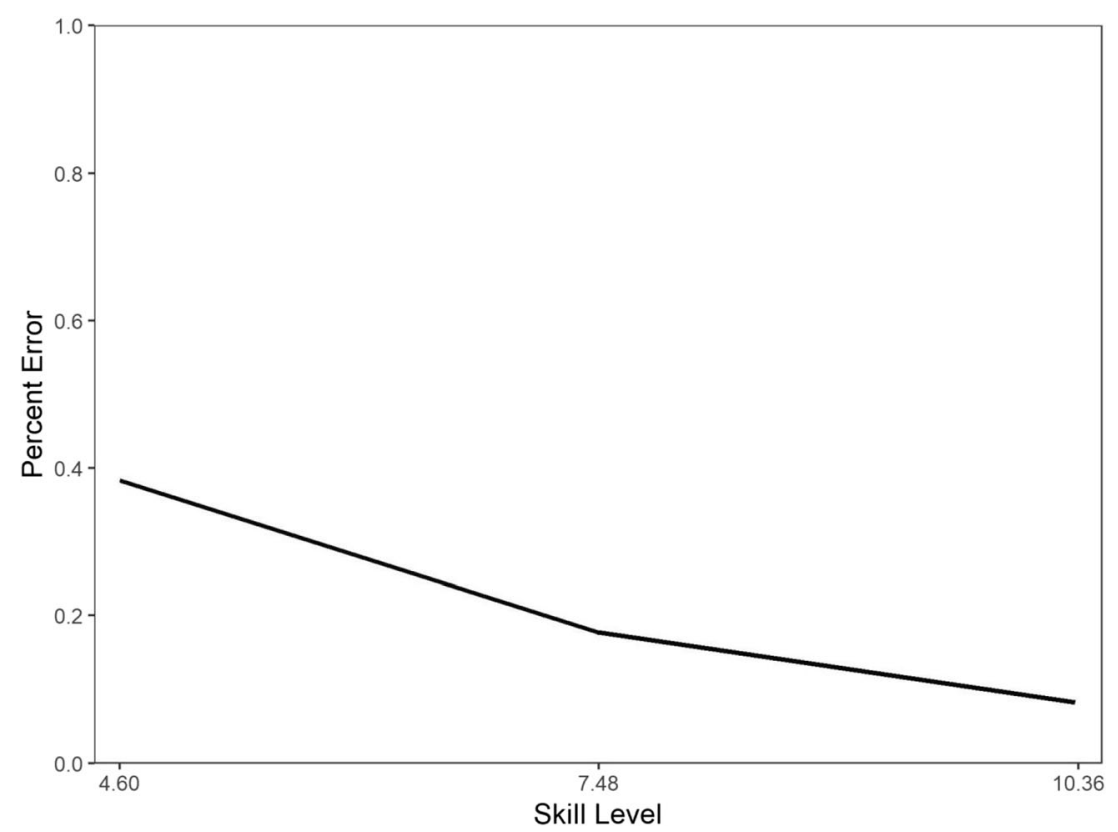

Fig. 7 The predicted sequence memory error rate for mean and $\pm 1 S D$ climbing skill after climbing. Values represent the rate (or percentage) of error for a single boulder route trial as a function of skill level 
Abernethy, 2003; Weast et al., 2011), and memory in experts (Abernethy et al., 1994; Allard et al., 1980; Charness, 1979; Chase \& Simon, 1973; Garland \& Barry, 1991; Reitman, 1976; Starkes, 1987; Ward \& Williams, 2003; Williams et al., 1993).

Understanding the mechanism for the expertise effects we found and how domain-specific skills are acquired over time requires further investigation. However, there are some theoretical concepts that may explain and contextualize our findings. The fact that we found that more skilled climbers have more accurate perceptual judgments and better visual and sequence memory provides some evidence to suggest that there is a link between what the observer perceives and what the observer remembers.

One explanation for the observed advantages for skilled climbers can be derived from the ecological approach (Gibson, 1979). This perspective would suggest that skilled climbers are more sensitive to the properties of their environment that specify affordances, which are generally defined as opportunities for action within a given environment perceived in terms of one's action capabilities (Gibson, 1979). Prior work has supported this claim with the finding that expert climbers attend more to the functional aspects (i.e., how holds can be grasped) compared with novices (Boschker et al., 2002). In other athletic domains, such as basketball and rugby, experience in the sport predicts more accurate assessment of domain-specific action capabilities (Correia et al., 2012; Weast et al., 2011). Therefore, the perceptual advantage we found in our first experiment could be a result of more tuned perceptual systems in skilled climbers. The ecological approach does include a mechanism for "memory" of actions (see Turvey \& Shaw, 1979), but only one study thus far has shown that affordances can be remembered even if the observer must recall properties of the environment that are not present (Wagman, Thomas, McBride, \& Day, 2013). Importantly, the previously mentioned study investigated the "memory" of singular affordances, and not a sequence of nested affordances (e.g., climbing move sequences). Therefore, it is unclear exactly how the ecological approach would explain advantages such as motor sequence memory or visual memory of holds.

Skilled climbers' ability to accurately perceive what their body is capable of and remember holds and sequences in a climbing environment could also be accounted for by the concept of motor simulations. Motor simulations are automatic and unconscious processes that take place in motor regions of the brain (e.g., supplementary motor area, inferior parietal lobe). Researchers have shown that such motor activations can be elicited by the sight of objects in the environment (Creem-Regehr \& Lee, 2005; Grafton, Fadiga, Arbib, \& Rizzolatti, 1997; Grèzes, Tucker, Armony, Ellis, \& Passingham, 2003; Tucker \& Ellis, 1998). These studies are particularly relevant to climbing because there is a rich variety of objects (holds) in a climbing environment and ways climbers can act on holds.

In the context of indoor rock climbing, motor simulations may be activated by the sight of holds on the climbing wall, but only if climbers are able to act upon them. This assumption is supported by findings that indicate the degree of motor activation in response to the sight of objects depends on the degree to which the observer can act or has been trained to act on the object (Creem-Regehr, Dilda, Vicchrilli, Federer, \& Lee, 2007). Previously, expertise differences in brain activation associated with motor simulation were seen for videos of dance sequences that look visually similar, but in which the physical lead up to movements as well as the completion of movements were different for particular styles of dance (Calvo-Merino, Glaser, Grèzes, Passingham, \& Haggard, 2004). Expertise in the specific dance style reflected knowledge of body movements that experts in the other dance styles and the nondancers did not have. The same may be true with climbers. It is difficult to know if more skilled climbers would simulate more actions in response to climbing holds or if all climbers simulate the same actions, but only experienced climbers are able to account for the nuances of the actions in their simulations. Regardless of the exact mechanism, we believe that motor simulation in skilled climbers could aid in their accurate action capability assessments.

The results of our second experiment can also be explained from a motor simulation perspective. Climbers that can simulate and combine multiple moves into a sequence could use each sequence as a meaningful unit by which to chunk routes. We inform this explanation via previous work that has shown chess experts' ability to organize board states into meaningful units of move sequences (Chase \& Simon, 1973). Our two main findings in Experiment 2, would support this explanation. First, we found that more skilled climbers had better visual memory in the preclimb phase. Therefore, more skilled climbers processed and encoded the information on the wall more effectively compared with less skilled climbers, prior to physical interaction with the holds. Less skilled climbers in our study may not have had the motor repertoire to fully engage a chunking mechanism, which likely constrained the amount of information they could remember given general working memory limits. Second, we found that climbing expertise positively related to one's performance on the motor sequence tasks before and after climbing the route. This indicates that while less skilled climbers' visual memory for holds improved after extended exposure to the routes, the exposure had no influence on the degree to which they were able to process information related to their actions.

\section{Limitations}

Certain limitations in the current study restrict the subsequent claims that can be made regarding our findings. We did not 
conduct a priori power analyses for either study. However, we confirmed that our main results in both experiments were robust with sensitivity analyses. In our first experiment, we found evidence to suggest that more skilled climbers are more likely to make underconfident errors, but our effect size did not fully meet the specified effect size of our sensitivity analysis. Consequently, interpretation of this finding is limited. We further acknowledge the limitations of this finding given problems with understanding and interpreting findings of underconfidence and overconfidence (Moore \& Healy, 2008).

In Experiment 2, our design was such that participants chose the routes they were tested on, in an attempt to scale for ability level., This also resulted in different routes for different participants, which may have confounded our results. For instance, participants selecting more difficult routes may have been able to identify holds on the selected route due to their size or spacing. While we believe this would be more likely to provide a benefit for those selecting easier routes due to the increased hold size, other possible confounds may exist that future research should more carefully control for. Further, participants were necessarily tested multiple times in Experiment 2. Since all the routes being tested on each individual climber were different, we were unable to control for order effects, and therefore do not know if the later tests were influenced by performing earlier tests (for example, in the form of practice effects). In order to mitigate this effect, all participants received a thorough explanation of the task before starting the first route of the experiment. However, further research would be required to determine whether participants developed strategies that led to advantages across trials.

Additionally, we propose motor simulation as an explaining mechanism behind our findings, but we did not gather direct evidence to support this claim. Previous brain imaging studies suggest that other motor experts (dancers) show more activity in the action-observation network when observing actions they are practiced in, compared with observing visually similar actions outside their domain (CalvoMerino et al., 2004). Our claim of motor simulation would predict a similar pattern of activation in rock climbers, but we did not gather imaging data to support this claim. Furthermore, we only tested indoor (not outdoor) rock climbers, so our results can only be reasonably generalized to an indoor rock-climbing population. Outdoor rockclimbing environments are even more variable given rock formations found in nature, and the conditions may be more anxiety provoking. Given this, results may differ in an outdoor setting. Because of the quasi-experimental nature of our study design, we also cannot eliminate the possibility that our findings were simply due to innate differences that existed before climbing experience was obtained. Further, we suggest that advantages in lower order perceptual skills are associated with advantages in higher order cognitive skills, but we did not directly test the relationship within a single task.

\section{Future directions}

Future research could evaluate perceptual and cognitive skills together by having climbers perform an affordance judgment task and a visual/sequence memory task within the same experiment. Researchers could also attempt to replicate the pattern of overconfidence and underconfidence found in our first experiment. To directly investigate motor simulation, researchers could examine if climbers shown objects that they could act upon (i.e., hold arrays), or climbers of different skill levels completing or attempting routes, reveal differential motor region activation among climbers of varying skill level, or nonclimber controls, using fMRI. Motor simulations could also be investigated behaviorally by suppressing gesturing (providing motor interference) during tasks similar to those in our experiments to examine if expertise effects were eliminated when gesturing is suppressed (Moreau, 2012; Smyth \& Pendleton, 1994). Furthermore, we found evidence to exclude the possibility that more skilled climbers have higher visuospatial working memory on a basic sequence memory task, but a more embodied or domain-relevant sequence task could be employed, such as the Barhorst twister task, to assess the extent to which sequence memory abilities in climbers might generalize (Barhorst-Cates, 2018).

\section{Conclusion}

In conclusion, our results provide evidence that expert climbers more accurately perceive what their body is capable of in a climbing environment, remember visual aspects (holds), and both plan and remember motor sequences for routes. Our results provide some evidence to suggest perceptual advantages as a result of motor expertise may underlie how individuals process and remember domain relevant information. This provides preliminary evidence that experts are more accurate at perceiving domain-related affordances, and this process may be related to their superior ability to remember visual aspects in their environment and the motor sequences they will perform. Future research is needed to disentangle explanations of our results and the link between perceptual and cognitive advantages in motor experts.

Acknowledgements This work was supported by the University of Utah Office of Undergraduate Research. Special thanks to the Summit Climbing Gym at the University of Utah Campus Recreation Center, and The Front Climbing Club. We would like thank Sarah CreemRegher and Ian Ruginski for feedback on earlier versions of this manuscript.

The data for Experiment 1 and 2 is available on Open Science Framework. 


\section{References}

Abernethy, B., Neal, R. J., \& Koning, P. (1994). Visual-perceptual and cognitive differences between expert, intermediate, and novice snooker players. Applied Cognitive Psychology, 8(3), 185-211.

Allard, F., Graham, S., \& Paarsalu, M. E. (1980). Perception in sport: Basketball. Journal of Sport Psychology, 2(1), 14-21.

Allard, F., \& Starkes, J. L. (1991). Motor-skill experts in sports, dance, and other domains. In K. A. Ericsson \& J. Smith (Eds.), Toward a general theory of expertise: Prospects and limits (pp. 126-152). Cambridge, England: Cambridge University Press.

Barhorst-Cates, E. M. (2018). Spatial working memory is enhanced for movement experts in traditional and embodied tasks. Spatial Cognition \& Computation, 1-23. Advance online publication. doi: https://doi.org/10.1080/13875868.2018.1541458

Boschker, M. S., Bakker, F. C., \& Michaels, C. F. (2002). Memory for the functional characteristics of climbing walls: Perceiving affordances. Journal of Motor Behavior, 34(1), 25-36.

Calvo-Merino, B., Glaser, D. E., Grèzes, J., Passingham, R. E., \& Haggard, P. (2004). Action observation and acquired motor skills: An FMRI study with expert dancers. Cerebral Cortex, 15(8), 12431249.

Charness, N. (1979). Components of skill in bridge. Canadian Journal of Psychology/Revue canadienne de psychologie, 33(1), 1-16.

Chase, W. G., \& Simon, H. A. (1973). Perception in chess. Cognitive Psychology, 4(1), 55-81.

Correia, V., Araújo, D., Cummins, A., \& Craig, C. M. (2012). Perceiving and acting upon spaces in a VR rugby task: Expertise effects in affordance detection and task achievement. Journal of Sport and Exercise Psychology, 34(3), 305-321.

Corsi, P. M. (1972). Human memory and the medial temporal region of the brain. Dissertation Abstracts International, 34(2-B), 891.

Cowan, N. (2010). The magical mystery four: How is working memory capacity limited, and why?. Current Directions in Psychological Science, 19(1), 51-57.

Creem-Regehr, S. H., Dilda, V., Vicchrilli, A. E., Federer, F., \& Lee, J. N. (2007). The influence of complex action knowledge on representations of novel graspable objects: Evidence from functional magnetic resonance imaging. Journal of the International Neuropsychological Society, 13(6), 1009-1020.

Creem-Regehr, S. H., \& Lee, J. N. (2005). Neural representations of graspable objects: Are tools special?. Cognitive Brain Research, 22(3), 457-469.

De Groot, A. D. (1965). Thought and choice in chess (Trans.). The Hague, Netherlands: Mouton.

Engle, R. W., \& Bukstel, L. (1978). Memory processes among bridge players of differing expertise. The American Journal of Psychology, 91(4), 673-689. doi:https://doi.org/10.2307/1421515

Farrow, D., \& Abernethy, B. (2003). Do expertise and the degree of perception-action coupling affect natural anticipatory performance? Perception, 32(9), 1127-1139.

Furley, P. A., \& Memmert, D. (2010). The role of working memory in sport. International Review of Sport and Exercise Psychology, 3(2), 171-194.

Garland, D. J., \& Barry, J. R. (1991). Cognitive advantage in sport: The nature of perceptual structures. The American Journal of Psychology, 104(2), 211-228.

Gibson, J. J. (1979). The ecological approach to visual perception. Boston, MA: Houghton Mifflin.

Grafton, S. T., Fadiga, L., Arbib, M. A., \& Rizzolatti, G. (1997). Premotor cortex activation during observation and naming of familiar tools. Neurolmage, 6(4), 231-236.

Green, A. L., Draper, N., \& Helton, W. S. (2014). The impact of fear words in a secondary task on complex motor performance: A dualtask climbing study. Psychological Research, 78(4), 557-565.
Green, A. L., \& Helton, W. S. (2011). Dual-task performance during a climbing traverse. Experimental Brain Research, 215(3/4), 307313.

Grèzes, J., Tucker, M., Armony, J., Ellis, R., \& Passingham, R. E. (2003). Objects automatically potentiate action: An fMRI study of implicit processing. European Journal of Neuroscience, 17(12), 2735-2740.

Kenny, D. A., Kashy, D. A., \& Cook, W. L. (2006). Dyadic data analysis. New York, NY: Guilford Press.

Kruger, J., \& Dunning, D. (1999). Unskilled and unaware of it: How difficulties in recognizing one's own incompetence lead to inflated self-assessments. Journal of Personality and Social Psychology, 77(6), 1121-1134. doi:https://doi.org/10.1037//0022-3514.77.6. 1121

Ma, W. J., Husain, M., \& Bays, P. M. (2014). Changing concepts of working memory. Nature Neuroscience, 17(3), 347-356.

Miller, G. A. (1956). The magical number seven, plus or minus two: Some limits on our capacity for processing information. Psychological Review, 63(2), 81-97.

Moore, D. A., \& Healy, P. J. (2008). The trouble with overconfidence. Psychological Review, 115(2), 502-517. doi:https://doi.org/10. 1037/0033-295X.115.2.502

Moreau, D. (2012). The role of motor processes in three-dimensional mental rotation: Shaping cognitive processing via sensorimotor experience. Learning and Individual Differences, 22(3), 354-359.

Nieuwenhuys, A., Pijpers, J. R., Oudejans, R. R., \& Bakker, F. C. (2008). The influence of anxiety on visual attention in climbing. Journal of Sport and Exercise Psychology, 30(2), 171-185.

Pereira, T., Abreu, A. M., \& Castro-Caldas, A. (2013). Understanding task-and expertise-specific motor acquisition and motor memory formation and consolidation. Perceptual and Motor Skills, 117(1), $108-129$.

Pezzulo, G., Barca, L., Bocconi, A. L., \& Borghi, A. M. (2010). When affordances climb into your mind: Advantages of motor simulation in a memory task performed by novice and expert rock climbers. Brain and Cognition, 73(1), 68-73.

Pijpers, J. R., \& Bakker, F. C. (1993). Perceiving affordances in climbing: Skill-effects. In S. S. Valenti \& J. B. Pittenger (Eds.), Studies in perception and action II: Posters presented at the VIIth International Conference on Event Perception and Action (pp. 8588). New York, NY: Academic Press.

Poulton, E. C. (1957). On prediction in skilled movements. Psychological Bulletin, 54(6), 467-478.

Raudenbush, S. W., \& Bryk, A. S. (2002). Advance Quantitative Techniques in the Social Sciences Series: Vol. 1. Hierarchical linear models: Applications and data analysis methods (2nd ed.). Thousand Oaks, CA: SAGE.

Raudenbush, S. W., Bryk, A. S., \& Congdon, R. (2011). HLM 7 for Windows [Computer software]. Skokie, IL: Scientific Software International.

Reitman, J. S. (1976). Skilled perception in Go: Deducing memory structures from inter-response times. Cognitive Psychology, 8(3), 336356.

Sherman, J., Head, M., Crump, J., \& Head, D. (1991). Heuco Tanks: A climber's and boulderer's guide. Guilford, CT: The Globe Pequot Press.

Smyth, M. M., \& Pendleton, L. R. (1994). Memory for movement in professional ballet dancers. International Journal of Sport Psychology, 25(3), 282-294.

Starkes, J. L. (1987). Skill in field hockey: The nature of the cognitive advantage. Journal of Sport Psychology, 9(2), 146-160.

Tucker, M., \& Ellis, R. (1998). On the relations between seen objects and components of potential actions. Journal of Experimental Psychology: Human Perception and Performance, 24(3), 830-846.

Turvey, M. T., Shaw, R., \& Nilsson, L. C. (1979). Perspectives on memory research. 
Voyer, D., \& Jansen, P. (2017). Motor expertise and performance in spatial tasks: A meta-analysis. Human Movement Science, 54, 110-124.

Wagman, J. B., Thomas, B. J., McBride, D. M., \& Day, B. M. (2013). Perception of maximum reaching height when the means of reaching are no longer in view. Ecological Psychology, 25(1), 63 80.

Ward, P., \& Williams, A. M. (2003). Perceptual and cognitive skill development in soccer: The multidimensional nature of expert performance. Journal of Sport and Exercise Psychology, 25(1), 93-111.

Weast, J. A., Shockley, K., \& Riley, M. A. (2011). The influence of athletic experience and kinematic information on skill-relevant affordance perception. The Quarterly Journal of Experimental Psychology, 64(4), 689-706.

Wickens, C. D., McLaughlin, A. C., Keller, J., \& Tan, J. (2018). Task switching in rock climbing: Validation of a computational model for different skill levels. Proceedings of the Human Factors and Ergonomics Society Annual Meeting, 61(1), 651-655.

Williams, M., Davids, K., Burwitz, L., \& Williams, J. (1993). Cognitive knowledge and soccer performance. Perceptual and Motor Skills, 76(2), 579-593.

Wright, E., Pinyan, E. C., Wickens, C. D., Keller, J., \& McLaughlin, A. C. (2018). Assessing dynamic value for safety gear during a rock climbing task. Proceedings of the Human Factors and Ergonomics Society Annual Meeting, 62(1), 1707-1711.

Publisher's note Springer Nature remains neutral with regard to jurisdictional claims in published maps and institutional affiliations. 